\title{
POSITIVE QUATERNIONIC KÄHLER MANIFOLDS AND SYMMETRY RANK
}

\author{
FUQUAN FANG ${ }^{1}$
}

\begin{abstract}
A quaternionic Kähler manifold $M$ is called positive if it has positive scalar curvature. The main purpose of this paper is to prove several connectedness theorems for quaternionic immersions in a quaternionic Kähler manifold, e.g. the Barth-Lefschetz type connectedness theorem for quaternionic submanifolds in a positive quaternionic Kähler manifold. As applications we prove that, among others, a $4 m$-dimensional positive quaternionic Kähler manifold with symmetry rank at least $(m-2)$ must be either isometric to $\mathbb{H} P^{m}$ or $G r_{2}\left(\mathbb{C}^{m+2}\right)$, if $m \geq 10$.
\end{abstract}

\section{InTRODUCTION}

A quaternionic Kähler manifold $M$ is an oriented Riemannian $4 n$-manifold, $n \geq$ 2, whose holonomy group is contained in $S p(n) S p(1) \subset S O(4 n)$. If $n=1$ we add the condition that $M$ is Einstein and self dual. Equivalently, there exists a 3-dimensional subbundle $S$, of the endmorphism bundle $\operatorname{End}(T M, T M)$ locally generated by three anti-commuting almost complex structures $I, J, K=I J$ so that the Levi-Civita connection preserves $S$. It is well-known [Ber] that a quaternionic Kähler manifold $M$ is always Einstein, and is necessarily locally hyperKähler if its Ricci tensor vanishes. Motivated by the Penrose twistor construction (cf. [AHS] [Hi]), Salamon [Sa] developped the important twistor space theory for quaternionic Kähler manifolds, showing that the unit sphere bundle of $S$, called the twistor space $Z$, admits a complex structure so that the fiber of the $\mathbb{P}^{1}$-fibration $p: Z \rightarrow M$ is a rational curve. A quaternionic Kähler manifold $M$ is called positive if it has positive scalar curvature. By [Hi] (for $n=1$ ) and [Sa] (for $n \geq 2$, compare [Le] [LeSa]) a positive quaternionic Kähler manifold $M$ has twistor space $Z$ a complex Fano manifold. Hitchin [Hi] proved a positive quaternionic Kähler 4-manifold $M$ must be isometric to $\mathbb{C} P^{2}$ or $S^{4}$. Hitchin's work was extended by Poon-Salamon [PS]

${ }^{1}$ Supported by NSF Grant 19925104 of China, 973 project of Foundation Science of China, RFDP

Typeset by $\mathcal{A} \mathcal{M} \mathcal{S}-\mathrm{T}_{\mathrm{E}} \mathrm{X}$ 
to dimension 8, showing that a positive quaternionic Kähler 8-manifold $M$ must be isometric to $\mathbb{H} P^{2}, G r_{2}\left(\mathbb{C}^{4}\right)$ or $G_{2} / S O(4)$. This leads to the Salamon-Lebrun conjecture:

Every positive quaternionic Kähler manifold is a quaternionic symmetric space.

Very recently, the conjecture was further verified for $n=3$ in [HH], using approach initiated in [Sa] [PS] (compare [LeSa]). For a positive quaternionic Kähler manifold $M$, Salamon [Sa] proved that the dimension of its isometry group is equal to the index of certain twisted Dirac operator, which by the Atiyah-Singer index theorem, is a characteristic number of $M$ coupled with the Kraines 4-form $\Omega$ (in analog with the Kähler form), and it was applied to prove the isometry group of $M$ is large in lower dimensions (up to dimension 16).

By [LeSa] any positive quaternionic Kähler $4 n$-manifold $M$ is simply connected and the second homotopy group $\pi_{2}(M)$ is a finite group or $\mathbb{Z}$, and $M$ is isometric to $\mathbb{H} P^{n}$ or $G r_{2}\left(\mathbb{C}^{n+2}\right)$ according to $\pi_{2}(M)=0$ or $\mathbb{Z}$.

The main purpose of this paper is to prove several connectedness theorems for positive quaternionic submanifolds, by using Morse theory on path spaces, e.g. the analog of the well-known Lefschetz hyperplane section theorem [Le] (cf. [FL] $[\mathrm{Fu}]$ ), Barth-Lefschetz connectedness theorem, among others. In particular, our results show that a quaternionic Kähler submanifold of small codimension in a positive quaternionic Kähler manifold shares the homotopy groups up to a certain range.

By Gray [Gr] any quaternionic Kähler submanifold must be totally geodesic, which often shows up as fixed point components of isometric actions. Our connectedness theorems may be applied to study positive quaternionic Kähler manifold in terms of informations on its isometry group. This approach dates back to the work [PS] for $n=2[\mathrm{HH}]$ for $n=3$ to proving the action is transitive, and [DS] [PV] for cohomogeneity one actions (and hence the isometry group must be very large). [Bi] classified positive quaternionic Kähler $4 n$-manifolds with isometry rank $n+1$, using an approach on hyper-Kähler quantizations. Based on our connectedness theorems we will prove that, using a much more direct and transparent approach, a positive quaternionic Kähler $4 n$-manifolds with isometry rank $\geq n-2$ must be either isometric to $\mathbb{H} P^{n}$ or $G r_{2}\left(\mathbb{C}^{n+2}\right)$, if $n \geq 10$.

For the sake of simplicity manifolds, $M, N_{1}, N_{2}$ in the paper, are all closed and 
connected manifolds. Let $M, N$ be quaternionic manifolds. An immersion $f: N \rightarrow$ $M$ is called quaternionic if $f$ preserves the quaternionic structures.

\section{Theorem A.}

Let $M$ be a positive quaternionic Kähler manifold of dimension 4m. Assume $f=\left(f_{1}, f_{2}\right): N \rightarrow M \times M$, where $N=N_{1} \times N_{2}$ and $f_{i}: N_{i} \rightarrow M$ are quaternionic immersions of compact quaternionic Kähler manifolds of dimensions $4 n_{i}, i=1,2$. Let $\Delta$ be the diagonal of $M \times M$. Set $n=n_{1}+n_{2}$. Then:

(A1) If $n \geq m$, then $f^{-1}(\Delta)$ is nonempty.

(A2) If $n \geq m+1$, then $f^{-1}(\Delta)$ is connected.

(A3) If $f$ is an embedding, then for $i \leq n-m$ there is a natural isomorphism, $\pi_{i}\left(N_{1}, N_{1} \cap N_{2}\right) \rightarrow \pi_{i}\left(M, N_{2}\right)$ and a surjection for $i=n-m+1$.

Some comments on Theorem A are in order.

First, (A1) implies immediately the following intersection theorem, which is due to Marchiafava [Ma] for embedded quaternionic Kähler submanifolds.

\section{Theorem 0.1.}

Let $M$ be a positive quaternionic Kähler manifold of dimension $4 m$. Let $f_{i}$ : $N_{i} \rightarrow M, i=1,2$, be two immersions of quaternionic Kähler manifolds. If $\operatorname{dim}\left(N_{1}\right)+\operatorname{dim}\left(N_{2}\right) \geq 4 m$, then $f_{1}\left(N_{1}\right) \cap f_{2}\left(N_{2}\right) \neq \emptyset$.

Secondly, (A2) implies the following regularity result.

\section{Theorem 0.2 (Regularity).}

Let $M$ be a positive quaternionic Kähler manifold of dimension $4 m$. If $f: N \rightarrow$ $M$ be a quaternionic immersion of a quaternionic Kähler manifold of dimension 4n. Then $f$ is an embedding, provided $2 n \geq m+1$.

Theorem 0.2 may be considered as a quaternionic analog of the well-known Fulton-Hansen immersion theorem $([\mathrm{FH}])$ for local complete intersections in $\mathbb{C} P^{m}$, which asserts that a finite unramified morphism from an algebraic variety of dimension $n$ to $\mathbb{C} P^{m}$ must be an embedding, if the dimension satisfies $2 n>m$.

Thirdly, (A3) is exactly the analog of the classical Barth-Lefschetz hyperplane section Theorems. A particular case implies that the analog of the Lefschetz the- 
orem, namely, the inclusion $i: N \rightarrow M$ is $(2 n-m+1)$-connected where $N$ is a quaternionic Kähler submanifold of dimension $4 n$.

(A3) is sharp, since $\operatorname{Gr}_{2}\left(\mathbb{C}^{4}\right)=\widetilde{\mathrm{Gr}}_{4}\left(\mathbb{R}^{6}\right) \subset \widetilde{\mathrm{Gr}_{4}}\left(\mathbb{R}^{7}\right)$ is a quaternionic Kähler submanifold, for which the inclusion is 2-connected but not 3-connected, because the Betti numbers $b_{2}\left(\operatorname{Gr}_{2}\left(\mathbb{C}^{4}\right)\right)=1$, and $b_{2}\left(\widetilde{G r}_{4}\left(\mathbb{R}^{7}\right)\right)=0$. In general, the natural inclusion $\widetilde{G r}_{4}\left(\mathbb{R}^{m}\right) \subset \widetilde{G r}_{4}\left(\mathbb{R}^{m+1}\right)$ is a quaternionic Kähler submanifold, for which (A3) can not be improved.

For a Riemannian manifold, by the symmetry rank we mean the rank of its isometry group. Bielawski [Bi] proved that a positive quaternionic Kähler 4mmanifold of symmetry rank at least $m+1$ is isometric to $\mathbb{H} P^{m}$ or $G r_{2}\left(\mathbb{C}^{m+2}\right)$. Now we state a rigidity theorem of positive quaternionic Kähler manifolds in terms its symmetry rank using (A3), which much enhances Bielawski's theorem.

\section{Theorem B.}

Let $M$ be a positive quaternionic Kähler manifold of dimension $4 m$. Then the isometry group Isom $(M)$ has rank (denoted by rank $(M))$ at most $(m+1)$, and $M$ is isometric to $\mathbb{H} P^{m}$ or $G r_{2}\left(\mathbb{C}^{m+2}\right)$ if $\operatorname{rank}(M) \geq m-2$ and $m \geq 10$.

The idea to of proving Theorem B is roughly as follows: First note that the $T^{r}$-action on $M$ must have non-empty fixed point set since the Euler characteristic $\chi(M)>0$ by [Sa]. Consider the isotropy representation of $T^{r}$ at a fixed point $x \in$ $M$, which must be a representation through the local linear holonomy $S p(n) S p(1)$ at $T_{x} M \cong \mathbb{H}^{n}$. Under the condition on $r$ we will prove that there are a chain of quaternionic Kähler submanifolds in $M$,

$$
M^{4} \subset M^{8} \subset M^{12} \cdots \subset M
$$

such that every nearby two manifolds have relatively small dimension difference, and the 12-dimensional quaternionic Kähler manifold $M^{12}$ has either symmetry rank 4 or an effective $T^{3}$-action of quaternionic type (see Section 2 for the definition). Combining $[\mathrm{HH}]$ we see that $M^{12}=\mathrm{Gr}_{2}\left(\mathbb{C}^{5}\right)$ or $\mathbb{H} P^{3}$. By Theorem (A3) we know that $\pi_{2}(M)$ must be isomorphic to $\pi_{2}\left(M^{12}\right)$ through the chain of quaternionic Kähler submanifolds. By now we apply [LeSa] to conclude the desired result. 
This paper was partially inspired by the work [FMR], where a connectedness principle was developped for minimal submanifolds in a positively curved manifold.

The rest of the paper is organized as follows:

In Section 1, we recall some preliminary about quaternionic Kähler manifolds.

In Section 2, we prove Theorem B assuming Theorem A.

In Section 3, we apply Morse theory to suitable path spaces.

In Section 4, we prove some indices Theorems for energy functions.

In Section 5, we prove Theorem A and its corollaries.

Acknowledgment: The author would like to thank S. Salamon for some comment on quaternionic Kähler manifolds, and to thank Universidade Federal Fluminense (UFF) for the hospitality during the preparation of this work.

\section{QuATERNIONIC KÄHLER MANIFOLDS}

In this section we recall some basic results on quaternionic Kähler manifolds needed in later sections.

Let $(M, g)$ be a connected Riemannian $4 n$-manifold, $n \geq 2$. We say that $(M, g)$ is a quaternionic Kähler manifold if the holonomy group is conjugate to a subgroup in $S p(n) S p(1)=S p(n) \times_{\mathbb{Z}_{2}} S p(1) \subset S O(4 n)$. In dimension 4 we say $(M, g)$ is quaternionic Kähler manifold if it is Einstein, with non-zero scalar curvature, and self dual. A quaternionic Kähler manifold is positive if it has positive scalar curvature.

The quaternionic projective spaces $\mathbb{H} P^{n}$, the complex Grassmannian $G r_{2}\left(\mathbb{C}^{n+2}\right)$,

and the oriented real Grassmannian $\widetilde{\mathrm{Gr}}_{4}\left(\mathbb{R}^{n+4}\right)$ are quaternionic Kähler manifolds with positive scalar curvature. By Wolf $[\mathrm{Wo}]$ there is exactly one quaternionic symmetric space for each compact simple Lie algebra, and by Alekseevskii [Al] every compact quaternion homogeneous space is a symmetric space.

As we mentioned in the introduction, by far quaternionic symmetric spaces are 
the only known examples of positive quaternionic Kähler manifold.

\section{Theorem 1.1 ([Le-Sa $]$ ).}

(i) (Fininteness) For any $n$, there are, modulo isometries and rescalings, only finitely many positive quaternionic Kähler 4n-manifolds.

(ii) (Strong rigidity) Let $(M, g)$ be a positive quaternionic Kähler 4n-manifold. Then $M$ is simply connected and

$$
\pi_{2}(M)=\left\{\begin{array}{l}
0,(M, g)=\mathbb{H} P^{n} \\
\mathbb{Z},(M, g)=G r_{2}\left(\mathbb{C}^{n+2}\right) \\
\text { finite with 2-torsion, otherwise }
\end{array}\right.
$$

The following curvature relations due to Berger [Be2] will be very useful.

Proposition 1.2 ([Ber]). Let $(M, g)$ be a quaternionic Kähler 4n-manifold with Einstein constant $\lambda$. Let $I, J, K$ be the locally defined three anticommuting almost complex structures. Then the curvature operator satisfies the following identities:

$$
\begin{aligned}
& \langle R(X, I X) X, I X\rangle+\langle R(X, J X) X, J X\rangle+\langle R(X, K X) X, K X\rangle=\frac{3 \lambda}{n+2}\|X\|^{4} \\
\langle R(X, Y) X, Y\rangle+\langle R(X, I Y) X, I Y\rangle+\langle R(X, J Y) X, J Y\rangle+\langle R(X, K Y) X, K Y\rangle= & \\
= & \frac{\lambda}{n+2}\|X\|^{2}\|Y\|^{2}
\end{aligned}
$$

Proposition 1.2 was used to prove a quaternionic Kähler manifold is Einstein [Ber].

Theorem 1.3 ([Fuj] [NT]). Let $M$ be a positive quaternionic Kähler manifold. Then the intersection form of $M$ is positive definite.

A submanifold $N$ in a quaternionic Kähler manifold is called a quaternionic submanifold if the quaternionic structure (i.e. locally defined $I, J, K$ ) preserves the tangent bundle of $N$.

Proposition 1.4 ([Gr]). Any quaternionic submanifold in a quaternionic Kähler manifold is totally geodesic and quaternionic Kählerian. 


\section{Symmetry Rank of Positive Quaternionic Kähler manifold}

Let $M$ be a positive quaternionic Kähler manifold of dimension $4 n$. We call the rank of the isometry group $\operatorname{Isom}(M)$ the symmetry rank of $M$, denoted by $\operatorname{rank}(M)$. Bielawski [Bi] obtained that a positive quaternionic Kähler manifold of symmetry rank $n+1$ is isometric to $\mathbb{H} P^{n}$ or $G r_{2}\left(\mathbb{C}^{n+2}\right)$, via studying hyperkähler $4 n+4$ manifolds with tri-Hamiltonian $\mathbb{R}^{n+1}$-action. In this section we prove Theorems $\mathrm{B}$ and C assuming Theorem A. Our proof of Theorem B also give a more transparent and direct proof of his result.

To start let us note that $\operatorname{rank}(M)=n+1$ if $M=\mathbb{H} P^{n}$ or $\operatorname{Gr}_{2}\left(\mathbb{C}^{n+2}\right)$, and $\operatorname{rank}(M)=\left[\frac{n}{2}\right]+2$ if $M=\widetilde{\mathrm{Gr}}_{4}\left(\mathbb{R}^{n+4}\right)$.

\section{a). A upper bound for symmetry rank.}

Let $G \subset \operatorname{Isom}(M)$ be a connected Lie group. For any $x \in M$, the isotropy subgroup $G_{x}$ is a subgroup of the holonomy group $(S p(n) S p(1))_{x}$ at $x$, by the Kostant's Theorem (compare [PS] page 366). Therefore the isotropy representation is constituted from the two homomorphisms

$$
\rho_{x}: G_{x} \rightarrow S p(n)_{x}, \quad \bar{\rho}_{x}: G_{x} \rightarrow S p(1)_{x}
$$

Identifying $T_{x} M \cong \mathbb{H}^{n}$ with a quaternionic structure from the right, observe that $S p(n)_{x}$ (resp. $S p(1)_{x}$ ) acts on $T_{x} M$ from the left quaternionically linearly (resp. the right by quaternion multiplication.)

Lemma 2.1. Let $M$ be a positive quaternionic Kähler manifold of dimension $4 n$. Then $\operatorname{rank}(M) \leq n+1$.

Proof. Let $r$ be the symmetry rank of $M$. Consider the action of $T^{r}$ on $M$. By $[\mathrm{Sa}]$ the Euler charateristic $\chi(M)>0$. Therefore the fixed point set of $T^{r}$ is not empty. Consider the isotropy representation of $T^{r}$ at $T_{x} M \cong \mathbb{H}^{n}$, where $x$ is a fixed point. Since $T^{r} \subset(S p(n) S p(1))_{x}$, so $r \leq \operatorname{rank}(S p(n) S p(1))=n+1$. The desired result follows.

Lemma 2.2. Let $M$ be a positive quaternionic Kähler manifold of dimension $4 n$ with symmetry rank $r$. Then there exists a positive quaternionic Kähler submanifold of $M$ of dimension at least $4(r-2)$. 
Proof. Let $x \in M$ be a fixed point of $T^{r}$. Consider the isotropy representation of $T^{r}$ at $T_{x} M$. Observe that $\rho_{x}\left(T^{r}\right)$ has rank at least $(r-1)$, since the rank of $S p(1)$ is 1 . Observe that $\rho_{x}\left(T^{r}\right)$ acts on $T_{x} M \cong \mathbb{H}^{n}$ quaternionically linearly. By linear algebra there is a circle subgroup $S^{1} \subset \rho_{x}\left(T^{r}\right)$ with fixed point set a quaternionic linear subspace of dimension at least $4(r-2)$. Let $N$ be the fixed point component of $S^{1}$ containing $x$. Clearly the tangent space $T_{x} N$ is the quaternionic subspace of $T_{x} M$ fixed by $S^{1}$. Since $N$ is totally geodesic (the fixed point set of an isometric action), for any $y \in N$ there exists a geodesic $\gamma$ in $N$ joining $x$ and $y=\gamma(1)$. For any vector $X \in T_{x} N$, consider the parallel vector field $X(t)$ along $\gamma$. By [Ma] Lemma 8.2 we may choose an open set $U$ containing $\gamma$ in $M$ so that the quaternionic structure $I, J, K$ are defined on $U$ and $I X(t), J X(t), K X(t)$ are all parallel along $\gamma$. Obviously $I X(1), J X(1), K X(1)$ belongs to $T_{y} N$. Therefore $T_{y} N$ is a quaternionic subspace of $T_{y} M$. This proves that $N$ is a quaternionic submanifold. By Prop. 1.2 and 1.4 $N$ is a positive quaternionic Kähler manifold.

Remark. It may not be true that any fixed point component of the above chosen circle subgroup is a quaternionic submanifold. For example, $\operatorname{Gr}_{2}\left(\mathbb{C}^{4}\right)$ admits an isometric circle action with fixed point set $\mathrm{Gr}_{2}\left(\mathbb{C}^{3}\right) \cup \mathrm{Gr}_{1}\left(\mathbb{C}^{3}\right)$, the former one is a quaternionic Kähler submanifold, but the latter is not (but Kähler). However, by the proof of Lemma 2.2 we may have a criterion, namely, if a fixed point component has dimension greater than the middle dimension $2 n$, then it must be a quaternionic submanifold.

An isometric $G$-action on $M$ is called of quaternionic type if $\bar{\rho}_{x}: G \rightarrow S p(1)_{x}$ is trivial for any $G$-fixed point $x$.

To make our proof more transparent, we now prove Bielawski's result using our approach, which is essentially included in the following proposition.

Proposition 2.3. Let $M$ be a positive quaternionic Kähler manifold of dimension $4 m$ with symmetry rank $r$. If either $r=m+1$ or $r=m$ and the $T^{m}$-action is of quaternionic type. Then $M=\mathbb{H} P^{m}$ or $G r_{2}\left(\mathbb{C}^{m+2}\right)$.

Proof. We use induction on the dimension.

By $[\mathrm{PS}][\mathrm{HH}]$ we may assume that $m \geq 4$. Observe that the fixed point set for the $T^{r}$-action must be isolated. Let $x \in M$ be a fixed point. By Lemma 2.2 (and 
its proof) there is a positive quaternionic Kähler $4(m-1)$-submanifold $N^{4(m-1)}$ containing $x$. Consider the induced $T^{r}$-action on $N^{4(m-1)}$. Its principal isotropy group has rank at most 2 , and has rank 1 if the $T^{r}$-action is of quaternionic type.

Case (i). The principal isotropy group has rank 1.

There is an effective quotient action of $T^{r} / S^{1}=T^{r-1}$ on $N^{4(m-1)}$, which is quaternionic type if $r=m$. By induction we may assume that $N^{4(m-1)}=\mathbb{H} P^{m-1}$ or $\mathrm{Gr}_{2}\left(\mathbb{C}^{m+1}\right)$. By Theorem A3 and Theorem 1.1 the desired result follows.

Case (ii). The principal isotropy group has rank 2 .

The principal isotropy group $T^{2} \subset T^{r}$ acts on the normal space of $T_{x} N^{4(m-1)}$ in $T_{x} M$, which may be identified with $\mathbb{H}$. If $\bar{\rho}_{x}: T^{2} \rightarrow S p(1)_{x}$ is trivial, $T^{2}$ acts on the normal space quaternionically linearly. For the dimension reason there is a circle subgroup of $T^{2}$ acting trivially on the normal space. This is impossible since the $T^{r}$-action is effective. In particular, we get that $r=m+1$.

Note that the isotropy representation of the principal isotropy group does not depend on the choice of the point in $N^{4(m-1)}$. Thus $\bar{\rho}_{x}: T^{2} \rightarrow S p(1)_{x}$ is nontrivial for any $x \in N^{4(m-1)}$. Note that $\bar{\rho}_{x}\left(T^{2}\right)$ acts trivially on $T_{x}\left(N^{4(m-1)}\right)$ and $\bar{\rho}_{x}\left(T^{r}\right)=$ $\bar{\rho}_{x}\left(T^{2}\right)$. Therefore the quotient action $T^{r} / T^{2}=T^{m-1}$ on $N^{4(m-1)}$ is of quaternionic type. By induction we may assume that $N^{4(m-1)}=\mathbb{H} P^{m-1}$ or $\operatorname{Gr}_{2}\left(\mathbb{C}^{m+1}\right)$. For the same reason as in Case (i) the desired result follows.

\section{b). Proof of Theorem B.}

We start with several lemmas.

Lemma 2.4. Let $T^{k}$ act effectively and quaternionically linearly on a quaternionic linear space $V \cong \mathbb{H}^{n}$ with fixed point set the origin $\{0\}$. Let $H_{1}, H_{2}, \cdots, H_{l}$ be the set of $(k-1)$-dimensional isotropy groups, and let $V_{1}, \cdots, V_{l}$ be the corresponding subspaces fixed by the isotropy groups. Let $n_{1}, \cdots, n_{l}$ be the $\mathbb{H}$-dimensions of $V_{1}, \cdots, V_{l}$. Then $l \geq k, V=V_{1}+\cdots+V_{l}$, and $n=n_{1}+\cdots+n_{l}$.

Proof. By Bredon [Br] III. Theorem 10.12 we know that $V=V_{1}+\cdots+V_{l}$ and $n=$ $n_{1}+\cdots+n_{l}$. It suffices to show that $l \geq k$. Suppose not, the intersection $H_{1} \cap \cdots \cap H_{l}$ is a non-trivial subgroup of $T^{k}$ which acts trivially on $V$. A contradiction with the effectiveness of the action. 
Lemma 2.5. Let $M$ be a positive quaternionic Kähler manifold of dimension $4 m$ with symmetry rank $r$. Assume $b_{2}(M)=0$. If $r \geq m-2 \geq 5$. Then the $T^{r}$-action on $M$ has at least an isolated fixed point.

Proof. Since the Euler characteristic $\chi(M)>0$ by [Sa], the fixed point set of $T^{r}$ is not empty. Choose a fixed point $x \in M$. Then $\rho_{x}: T^{r} \rightarrow S p(m)$ has an image of rank at least $r-1$. Let $N$ be the fixed point component of $\rho_{x}\left(T^{r}\right)$ containing $x$. By Lemma $2.2 N$ is a quaternionic Kähler submanifold of dimension $4 i$. Note that $4 i+4(r-1) \leq 4 m$. Thus $i \leq 3$. Clearly we may assume $i \geq 1$.

Consider the isotropy representation of $\rho_{x}\left(T^{r}\right)$ at the normal vector space of $T_{x} N \subset T_{x} M$. By Lemma 2.4 and the assumptions we may assume two quaternion subspaces $V_{1}, V_{2}$ of dimensions 4 with codimension one isotropy groups $H_{1}, H_{2} \subset$ $\rho_{x}\left(T^{r}\right)$. Note that $V_{1}+V_{2}+T_{x} N$ is the tangent space of the fixed point component at $x$ of the action of $H_{1} \cap H_{2}$, saying $N_{2}$. Note that $N \subset N_{2}$ has codimension 8 which extends to a chain of quaternionic Kähler submanifolds $N \subset N_{1} \subset N_{2}$ of codimensions 4 .

Case (i). If $i=1$;

Consider the restricted $\rho_{x}\left(T^{r}\right)$-action on the stratum $N_{2}$. By the construction $\rho_{x}\left(T^{r}\right)$ acts on $T_{x} N_{2}$ with principal isotropy group a codimension 2 subtorus. Since $\operatorname{dim} N_{2}=12$, by $[\mathrm{HH}]$ we know that $N_{2}=\mathbb{H} P^{3}, \widetilde{G r}_{4}\left(\mathbb{R}^{7}\right)$ or $\operatorname{Gr}_{2}\left(\mathbb{C}^{5}\right)$. By Theorem (A3) $b_{2}\left(N_{2}\right)=b_{2}(M)=0$. Thus we need only to consider the former two cases. If $N_{2}=\mathbb{H} P^{3}$ it is obvious. Now we assume that $N_{2}=\widetilde{\mathrm{Gr}}_{4}\left(\mathbb{R}^{7}\right)$. By [PS] we know that the quaternionic Kähler submanifold $N_{1}$ must be $\mathbb{H} P^{2}, G_{2} / S O(4)$ or $\mathrm{Gr}_{2}\left(\mathbb{C}^{4}\right)$. Since the $\rho_{x}\left(T^{r}\right)$-action on $\widetilde{\mathrm{Gr}}_{4}\left(\mathbb{R}^{7}\right)$ is a subaction of the standard transitive $S O(7)$ action, one sees easily that any circle action can not have an 8-dimensional fixed point component which is quaternionic. A contradiction.

Case (ii). If $i=2$;

Note that $\operatorname{dim} N=8$ and $\operatorname{dim} N_{1}=12$. Then $\rho_{x}\left(T^{r}\right)$ acts on $N_{1}$ with principal isotropy group rank codimension 1 . As above we may assume that $N_{1}=\mathbb{H} P^{3}$ or $\widetilde{\mathrm{Gr}}_{4}\left(\mathbb{R}^{7}\right)$. For the same reason as in Case (i) we see that $N_{1}=\mathbb{H} P^{3}$. The desired result follows.

Case (iii). If $i=3$; 
By (i) and (ii) we may assume that at every fixed point of $T^{r}$, quaternionic Kähler submanifolds passing through $x$ fixed by some toric subgroups, have dimensions at least 12. Note that $\operatorname{sig}(N)=0$ since $\operatorname{dim} N=12$ by $[\mathrm{HH}]$. As the Kraines' form $\Omega$ satisfies $\Omega^{2} \neq 0 \in H^{8}\left(N_{1}\right)$, we see that $b_{8}\left(N_{1}\right) \geq 1$. By Theorem 1.3 $\operatorname{sig}\left(N_{1}\right)=b_{8}\left(N_{1}\right)>0$. By [HS] we know that the $T^{r}$-action has a fixed point $y \in N_{1}-N$.

Consider the isotropy representation of $T^{r}$ on the normal vector space of $T_{y} N_{1}$. Let $H$ be the isotropy subgroup of the linear action on $T_{y} N_{1}$ (i.e. the principal isotropy group of $N_{1}$ ). It is easy to see that $H$ has rank at most $m-3$. Moreover, if $H$ has rank $m-3$, then $\bar{\rho}_{y}: H \subset T^{r} \rightarrow S p(1)$ is non-trivial and the restricted $T^{r}$ action on $N_{1}$ is quaternionic type. If $y$ is not an isolated fixed point, we may assume that $T^{r} / H$ has rank 1 and the $T^{r} / H$-action on $N_{1}$ is quaternionic type (otherwise $T^{r} / H$ may be replaced by a rank one subgroup which is quaternionic type at $y$ ). We see that $T^{r} / H$ has a fixed point component passing through $y$, which is a quaternionic Kähler submanifold in $N_{1}$ of dimension $\geq 4$. By Theorem 0.1 this quaternionic Kähler submanifold must intersect with $N$, which is clearly impossible. By now the proof is complete.

Lemma 2.6. Let $T^{r}$ act effectively and isometrically on a quaternionic Kähler manifold $M^{4 m}$ with an isolated fixed point $x$. If $r \geq \frac{m}{2}+3$, then there is a chain of quaternionic Kähler submanifolds

$$
x \subset N^{4} \subset N^{8} \subset N^{12} \subset \cdots N^{4 i} \subset N^{4 j} \subset \cdots \subset N^{4 s} \subset M^{4 m}
$$

such that $s \geq r-2$ and the dimensions $4 i, 4 j$ of every two nearby submanifolds in the chain satisfies that $2 i-j \geq 2$ for $i \geq 3$.

Proof. Consider the isotropy representation of $\rho_{x}\left(T^{r}\right)$ at the tangent space $T_{x} M \cong$ $\mathbb{H}^{m}$. By Lemma 2.4 we get $T_{x} M=V_{1}+\cdots+V_{l}$ where $l \geq r-1 \geq \frac{m}{2}+2$ and $V_{i}$ are all quaternionic subspaces. For the sake of simplicity, let $V_{1}, \cdots, V_{i_{1}}$ be the collection of subspaces of dimension 4. By Lemma $2.4 i_{1} \geq 4$ since $r-1 \geq \frac{m}{2}+2$. Let $V_{i_{1}+1}, \cdots, V_{i_{1}+i_{2}}$ be the collection of subspaces of dimension 8, etc.

If $i_{1} \geq r-2$, consider the sum $V_{1}+\cdots+V_{r-2}$. Observe that $H_{1} \cap \cdots \cap H_{r-2} \subset$ $\rho_{x}\left(T^{r}\right)$ has rank at least 1 . The fixed point component at $x$ of $H_{1} \cap \cdots \cap H_{r-2}$ is a quaternionic Kähler submanifold of $M$, saying $N^{4 r-8}$, of dimension $4 r-8$ whose 
tangent space at $x$ is $V_{1}+\cdots+V_{r-2}$. Clearly the desired chain of submanifolds may be obtained in a similar manner so that their tangent spaces at $x$ are given by a partial sum $V_{1}+\cdots+V_{i}$ for $i<r-2$.

If $i_{1}<r-2$, by Lemma $2.4 i_{2} \geq 1$ and $m \geq i_{1}+2 i_{2}+3 i_{3}+\cdots+k i_{k}$, where $4 i_{k}=$ $\max \left\{\operatorname{dim} V_{i}: 1 \leq i \leq l\right\}$. By Lemma $2.4 i_{1}+i_{2}+\cdots+i_{k} \geq r-1$. If $i_{1}+i_{2} \geq r-2$, choose the quaternionic Kähler submanifold $N^{4 i_{1}+8\left(r-2-i_{1}\right)}$ whose tangent space at $x$ is the sum $V_{1}+\cdots+V_{i_{1}}+V_{i_{1}+1} \cdots+V_{r-2-i_{1}}$, which has dimension $>4 r-8$. Otherwise we may proceed this inductively, assuming $i_{1}+i_{2}+\cdots+i_{k-1}<r-1$, then we may take the desired quaternionic Kähler submanifold $N^{4 i_{1}+8\left(r-2-i_{1}\right)}$ whose tangent space at $x$ is the sum $V_{1}+\cdots+V_{i_{1}}+\cdots+V_{i_{1}+\cdots+i_{k-1}}+\cdots+V_{r-2-i_{1}-\cdots-i_{k-1}}$. We may choose the quaternionic Kähler submanifolds of $N^{4 i_{1}+8\left(r-2-i_{1}\right)}$ for the chain so that their tangent spaces at $x$ are the partial sums as above. It is easy to verify that $2 i-j \geq 2$ from the construction.

Now we are ready to prove Theorem B.

\section{Proof of Theorem B.}

By Theorem 1.1 we may assume that $b_{2}(M)=0$. By Lemma 2.5 we may assume an isolated fixed point $x \in M$ of the $T^{r}$-action.

Consider the chain of quaternionic Kähler submanifolds defined in Lemma 2.6. Consider the restricted $T^{r}$-action on $N^{12}$. By the construction the principal isotropy group has rank $(r-4)$ or $(r-3)$. In the former case, there is an effective quotient action of $T^{4}$ on $N^{12}$. In the latter case we claim that the $T^{r}$-action on $N^{12}$ is quaternionic type. Assuming this, by Proposition $2.3 N^{12}=\mathbb{H} P^{3}$ or $\operatorname{Gr}_{2}\left(\mathbb{C}^{5}\right)$. Therefore by Theorem (A3) and Theorem 1.1 the desired result follows.

Clearly we need only to consider the case when $\bar{\rho}_{x}: T^{r} \rightarrow S p(1)_{x}$ is non-trivial. Since $T^{r} / T^{r-3}$ acts quaternionically linearly on $T_{x}\left(N^{12}\right) \cong \mathbb{H}^{3}$, the restricted representation of the isotropy group $T^{r-3} \subset T^{r} \stackrel{\bar{\rho}_{x}}{\longrightarrow} S p(1)_{x}$ is not trivial. Therefore, for any $y \in N^{12}$, the representation of the isotropy group $T^{r-3} \subset T^{r} \stackrel{\bar{\rho}_{y}}{\longrightarrow} S p(1)_{y}$ is not trivial. This implies readily that the $T^{r}$-action on $N^{12}$ is quaternionic type. 


\section{Morse Theory on Path Spaces}

Let $M$ be a complete Riemannian manifold without boundary and let $f: N \rightarrow$ $M \times M$ be an immersed complete submanifold of dimension $n$.

A piecewise smooth path in $M(\bmod f)$ is a pair $(x, \gamma)$, where $x \in N$ and a map $\gamma:[0,1] \rightarrow M$ such that:

(i) there is a subdivision $0=t_{0}<t_{1}<\cdots<t_{k}=1$ of $[0,1]$ such that each $\left.\gamma\right|_{\left[t_{i-1}, t_{i}\right]}$ is smooth.

(ii) $(\gamma(0), \gamma(1))=f(x)$.

The set of all piecewise smooth paths $(\bmod f)$ is denoted by $P(M ; f)$.

The topology of $P(M ; f)$ is taken the induced topology from $N \times P(M)$, where $P(M)$ is the space of piecewise smooth paths with the metric topology given by

$$
d\left(\gamma_{0}, \gamma_{1}\right)=\left\{\int_{0}^{1}\left(\left|\dot{\gamma}_{0}(t)\right|-\left|\dot{\gamma}_{1}(t)\right|\right)^{2} d t\right\}^{\frac{1}{2}}+\max _{0 \leq t \leq 1} d_{M}\left(\gamma_{0}(t), \gamma_{1}(t)\right) .
$$

Note that the integral is well-defined though $\dot{\gamma}_{i}(i=1,2)$ may not be defined at finitely many points in $[0,1]$.

On the space $P(M ; f)$ there is an energy function $E: P(M ; f) \rightarrow \mathbb{R}$ given by

$$
E(x, \gamma)=\int_{0}^{1}|\dot{\gamma}(t)|^{2} d t
$$

We want to study the topology of $P(M ; f)$ using Morse theory for the function $E$. When $f$ is an embedding, path space with this general boundary condition was studied in [Gr] using Morse theory on Hilbert manifolds. Instead, we will use the finite dimensional approximation methods to reduce to Morse theory in finite dimension.

The tangent space of $P(M ; f)$ at $(x, \gamma)$ is defined as the vector space of piecewise smooth vector fields $W$ along $\gamma$ such that $(W(0), W(1)) \in f_{*}\left(T_{x} N\right)$.

By a standard calculation the first variation of $E$ in the direction $W \in T_{\gamma} P(M, f)$, is given by

$$
\begin{aligned}
\frac{1}{2} E_{*}(W)= & -\int_{0}^{1}\left\langle W(t), \frac{D}{d t} \dot{\gamma}(t)\right\rangle d t+ \\
& \langle W(1), \dot{\gamma}(1)\rangle-\langle W(0), \dot{\gamma}(0)\rangle+\sum_{i=1}^{k-1}\left\langle W\left(t_{i}\right), \dot{\gamma}_{-}\left(t_{i}\right)-\dot{\gamma}_{+}\left(t_{i}\right)\right\rangle,
\end{aligned}
$$


where $\dot{\gamma}_{-}$is the left derivative and $\dot{\gamma}_{+}$is the right derivative of $\gamma$, and $\frac{D}{d t}$ is the covariant derivative along $\gamma$. So if $(x, \gamma)$ is a critical point of $E$ then:

(i) $\gamma$ is a smooth geodesic;

(ii) $(\dot{\gamma}(0),-\dot{\gamma}(1))$ is perpendicular to $f_{*}\left(T_{x}(N)\right)$ at $f(x)=(\gamma(0), \gamma(1))$.

Let $W_{1}, W_{2} \in T_{\gamma} P(M ; f)$. If $\gamma$ is a critical point of $E$ we consider any variation $h(t, s, u)$ of $\gamma$ with $\frac{\partial h}{\partial s}(t, 0,0)=W_{1}(t), \frac{\partial h}{\partial u}(t, 0,0)=W_{2}(t)$. Then the second variation of $E$ along $\gamma$, denoted by $E_{* *}\left(W_{1}, W_{2}\right)$, is as follows:

$$
\begin{aligned}
\frac{1}{2} E_{* *}\left(W_{1}, W_{2}\right)= & \int_{0}^{1}\left\{\left\langle\frac{D W_{1}}{d t}, \frac{D W_{2}}{d t}\right\rangle-\left\langle R\left(\dot{\gamma}, W_{1}\right) W_{2}, \dot{\gamma}\right\rangle\right\}+ \\
& \left\langle\frac{D}{d s} \frac{\partial h}{d u}(1,0,0), \dot{\gamma}(1)\right\rangle-\left\langle\frac{D}{d s} \frac{\partial h}{d u}(0,0,0), \dot{\gamma}(0)\right\rangle \\
= & \int_{0}^{1}\left\{\left\langle\frac{D W_{1}}{d t}, \frac{D W_{2}}{d t}\right\rangle-\left\langle R\left(\dot{\gamma}, W_{1}\right) W_{2}, \dot{\gamma}\right\rangle\right\}+ \\
& \left\langle\alpha\left(\left(W_{1}(0), W_{1}(1)\right),\left(W_{2}(0), W_{2}(1)\right)\right),(-\dot{\gamma}(0), \dot{\gamma}(1))\right\rangle,
\end{aligned}
$$

where $\alpha$ is the second fundamental form of the immersion $f: N \rightarrow M \times M$.

Let $P_{c}(M ; f)=E^{-1}([0, c)) \subset P(M ; f)$. Following Milnor-Morse we define a finite dimensional approximation to $P_{c}(M ; f)$ as follows:

Choose some subdivision $0=t_{0}<t_{1}<\cdots<t_{k}=1$ of $[0,1]$. Let $B$ be the subspace of $P_{c}(M ; f)$ such that

(i) $f(x)=(\gamma(0), \gamma(1))$

(ii) $\left.\gamma\right|_{\left[t_{i-1}, t_{i}\right]}$ is a geodesic for each $i=1, \cdots, k$.

Theorem 3.1. Let $M$ be a complete Riemannian manifold, and $f: N \rightarrow M \times M$ be an immersion where $N$ is a closed Riemannian manifold. Let c be a fixed positive number such that $P_{c}(M ; f)$ is not empty. Then for all sufficiently fine subdivision $0=t_{0}<t_{1}<\cdots<t_{k}=1$ of $[0,1]$ the set $B$ can be given the structure of a smooth finite dimensional manifold.

Proof. [Mi], Sect. 16.

Let $\left.E\right|_{B}: B \rightarrow \mathbb{R}$ be the restriction of the energy function $E$.

Theorem 3.2. Let $f, N, M$ be as in Theorem 2.1. Then $\left.E\right|_{B}$ is a smooth function. For each $a<c$ the set $\left(\left.E\right|_{B}\right)^{-1}([0, a])$ is compact, and $\left(\left.E\right|_{B}\right)^{-1}([0, a))$ is a deformation retract of the set $P_{a}(M ; f)$. The critical points of $\left.E\right|_{B}$ are precisely the same as the critical points of $E$ in $P_{c}(M ; f)$, that is, the pairs $(x, \gamma)$, where $\gamma$ 
is a smooth geodesic in $M$ such that $(\dot{\gamma}(0),-\dot{\gamma}(1))$ is normal to $f_{*}\left(T_{x}(N)\right)$ and the energy is less than $c$. The Hessians of $\left.E\right|_{B}$ and $\left.E\right|_{P_{c}(M ; f)}$ have the same index at each critical point $(x, \gamma)$.

Proof. [Mi], Sect. 14 and Sect. 16.

Lemma 3.3. Suppose that every nontrivial critical point $(x, \gamma)$ of $E$ has positive index. Then $f^{-1}(\Delta)$ is not empty.

Proof. Suppose $f^{-1}(\Delta)=\emptyset$. Writing $f(x)=\left(f_{1}(x), f_{2}(x)\right)$, from the compactness of $N$ we conclude that there exists $x \in N$ such that $d\left(f_{1}(x), f_{2}(x)\right)=\delta$ is a positive minimum. In particular $P(M ; f)$ contains no constant path and the energy function on $P(M ; f)$ assumes a positive minimum $\delta^{2}$ at $(x, \gamma)$, where $\gamma$ is a minimal geodesic joining $f_{1}(x)$ and $f_{2}(x)$. For this minimal critical point $(x, \gamma)$ the index is clearly zero. A contradiction to the assumption.

We also need the following lemma of Milnor for finite dimensional manifolds.

Lemma 3.4. Let $X$ be a finite dimensional smooth manifold and $f: X \rightarrow \mathbb{R}$ be a real function with minimal value zero. Suppose that for any a the sublevel set $X_{\leq a}=f^{-1}([0, a])$ is compact. Assume that the set $X_{0}$ of minimal points has a neighborhood $U$ with a deformation retraction $r: U \rightarrow X_{0}$, and that all nontrivial critical points have indices greater than $\lambda_{0} \geq 0$. Then $X$ has the homotopy-type of a $C W$-complex by attaching cells of dimensions at least $\lambda_{0}+1$ to $X_{0}$. In particular, $\pi_{j}\left(X, X_{0}\right)=0$ for $0 \leq j \leq \lambda_{0}$.

Proof. [Mi], Sect. 22.

Theorem 3.5. Let $M, N_{i}, i=1,2$, be compact quaternionic Kähler manifolds and $\Delta$ be the diagonal of $M \times M$. Let $f_{i}: N_{i} \rightarrow M$ be quaternionic immersions. Let $f=\left(f_{1}, f_{2}\right)$. Let $P_{0}=f^{-1}(\Delta)$. If every nontrivial critical point of $E$ on $P(M ; f)$ has index $\lambda>\lambda_{0} \geq 0$, then $P(M ; f)$ has the homotopy-type of a $C W$-complex obtained by attaching cells of dimensions at least $\lambda_{0}+1$ to $P_{0}$. In particular, the relative homotopy groups $\pi_{j}\left(P(M ; f), P_{0}\right)=0$ for $0 \leq j \leq \lambda_{0}$.

Proof. Note that each point $x$ in $P_{0}$ could be associated with a constant path at $p_{1} f(x)\left(=p_{2} f(x)\right)$. So $P_{0}$ can be identified with the constant geodesics in $P(M ; f)$. 
It suffices to prove that for any large value $c, P_{c}(M, f)$ has the homotopy-type of a CW-complex obtained by attaching cells of dimensions at least $\lambda_{0}+1$ to $P_{0}$. By Theorem $3.2 P_{c}(M, f)$ deformation retracts to $B$. Moreover, the index for every nontrivial critical point for the restricted energy function is greater than $\lambda_{0}$ too. The space $P_{0}$ is clearly inside $B$. To apply Lemma 3.4 it suffices to prove that there is a neighborhood $U \subset B$ of $P_{0}$ and a retraction $r: U \rightarrow P_{0}$.

Since a quaternionic immersion has to totally geodesic (cf. Prop. 1.4), the minimal set $P_{0}$ (resp. $\left.B\right)$ may be identified with the submanifold $\{(x, f(x), \cdots, f(x))$ : $\left.x \in f^{-1}(\Delta)\right\}$ (resp. an open submanifold of $\left.N \times(M \times M) \times \cdots \times(M \times M)\right)$. Therefore an open regular neighborhood of this submanifold is a desired open neighborhood $U$. The desired result follows.

\section{Indices TheOrems For CRItical points of Energy Functions}

Let $V$ be a $4 l$-dimensional quaternionic linear space and let $Q$ be a real symmetric bilinear form on $V$. Recall that the index of $Q$ is defined by the dimension of a maximal linear subspace $U \subset V$ so that $\left.Q\right|_{U}$ is negative definite.

Lemma 4.1. Let $V$ be a 4l-dimensional quaternionic linear space with invariant inner product $\langle.,$.$\rangle (i.e. I, J, K=I J$ preserve the inner product.) Let $Q(.,$.$) be a$ real symmetric bilinear form on $V$. Assume that for all nonzero vector $X \in V$ it holds that

$$
Q(X, X)+Q(I X, I X)+Q(J X, J X)+Q(K X, K X)<0
$$

Then the index $\lambda$ of $Q$ satisfies $\lambda \geq l$.

Proof. Let $A: V \rightarrow V$ be the symmetric linear map uniquely determined by $\langle A v, w\rangle=Q(v, w)$ for $v, w \in V$. Let $\mathcal{B}$ be an orthonormal basis of eigenvectors of the linear map $A: V \rightarrow V$. Consider the linear subspace $W$ spanned by the vectors $v \in \mathcal{B}$ with $Q(v, v) \geq 0$. Observe that the index of $Q$ is equal to $4 l-\operatorname{dim}(W)$. Therefore it suffices to prove that $\operatorname{dim}(W) \leq 3 l$. Suppose not, then the intersection $W \cap I W \cap J W \cap K W$ is a nontrivial linear subspace. For any nontrivial $v_{0}=I v_{1}=J v_{2}=K v_{3} \in W \cap I W \cap J W \cap K W$, where $v_{i} \in W$, we have $Q\left(v_{i}, v_{i}\right) \geq 0$. Therefore $Q\left(v_{0}, v_{0}\right)+Q\left(I v_{0}, I v_{0}\right)+Q\left(J v_{0}, J v_{0}\right)+Q\left(K v_{0}, K v_{0}\right) \geq 0$. A contradiction. The desired result follows. 
Let $M, N_{i}, i=1,2$, be compact quaternionic Kähler $4 m$-manifolds. Let $N=$ $N_{1} \times N_{2}$, and let $f_{i}: N_{i} \rightarrow M$ be quaternionic immersions. Set $f=\left(f_{1}, f_{2}\right)$ and $4 n=4 n_{1}+4 n_{2}$ for the real dimension of $N$. Let $W_{1}, W_{2} \in T_{(x, \gamma)} P(M ; f)$ be tangent vectors at $(x, \gamma)$. Let $\alpha$ be the second fundamental form of $f$ in $M \times M$. Recall that the second variation of the energy function $E$ along a critical point $(x, \gamma)$ reads

$$
\begin{aligned}
\frac{1}{2} E_{* *}\left(W_{1}, W_{2}\right)= & \int_{0}^{1}\left\{\left\langle\frac{D W_{1}}{d t}, \frac{D W_{2}}{d t}\right\rangle-\left\langle R\left(\dot{\gamma}, W_{1}\right) W_{2}, \dot{\gamma}\right\rangle\right\}+ \\
& \left\langle\alpha\left(\left(W_{1}(0), W_{1}(1)\right),\left(W_{2}(0), W_{2}(1)\right)\right),(-\dot{\gamma}(0), \dot{\gamma}(1))\right\rangle .
\end{aligned}
$$

Now let $W$ be parallel along $\gamma$ with $\mathcal{W}=(W(0), W(1)) \in f_{*}\left(T_{x} N\right)$. By [Ma] Lemma 8.2 we may assume that $I \mathcal{W}, J \mathcal{W}$ and $K \mathcal{W}$ are all parallel and tangent to $N$ at $x$, since $f_{i}$ are quaternionic immersions. Set

$$
\eta=(-\dot{\gamma}(0), \dot{\gamma}(1))
$$

Since $(x, \gamma)$ is a critical point, $(\dot{\gamma}(0),-\dot{\gamma}(1))$ is orthogonal to $\mathcal{W}, I \mathcal{W}, J \mathcal{W}$ and $K \mathcal{W}$. By the formula (4.2) we get

$$
\begin{aligned}
& \frac{1}{2} E_{* *}(W, W)+\frac{1}{2} E_{* *}(I W, I W)+\frac{1}{2} E_{* *}(J W, J W)+\frac{1}{2} E_{* *}(K W, K W)= \\
& =\int_{0}^{1}-\{\langle R(\dot{\gamma}, W) W, \dot{\gamma}\rangle+\langle R(\dot{\gamma}, I W) I W, \dot{\gamma}\rangle+\langle R(\dot{\gamma}, J W) J W, \dot{\gamma}\rangle+\langle R(\dot{\gamma}, K W) K W, \dot{\gamma}\rangle\}
\end{aligned}
$$

Let $\mathcal{V}$ be the real linear space spanned by vectors $(W(0), W(1))$, where $W$ is a parallel vector field along $\gamma$ so that $W$ orthogonal to $\dot{\gamma}, I \dot{\gamma}, J \dot{\gamma}$ and $K \dot{\gamma}$. The quaternionic dimension of $\mathcal{V}$ is $m-1$. If $\mathcal{W}=(W(0), W(1)) \in \mathcal{V}$ then $I \mathcal{W}, J \mathcal{W}$ and $K \mathcal{W} \in \mathcal{V}$ and $\mathcal{W}, I \mathcal{W}, J \mathcal{W}$, and $K \mathcal{W}$ are all orthogonal to $\eta=(-\dot{\gamma}(0), \dot{\gamma}(1))$, $I \eta, J \eta$ and $K \eta$. Therefore the quaternionic dimension

$$
\operatorname{dim}_{\mathbb{H}}\left(\mathcal{V} \cap f_{*}\left(T_{x} N\right)\right) \geq m-1+n-(2 m-1)=n-m
$$

Recall that if $\mathcal{W} \in \mathcal{V} \cap f_{*}\left(T_{x} N\right)$ then $W$ is a tangent vector of $P(M, f)$ at $(x, \gamma)$.

Theorem 4.2. Let $M$ be a positive quaternionic Kähler manifold of dimension 4m. Let $f_{i}: N_{i} \rightarrow M, i=1,2$, be quaternionic immersions of closed quaternionic Kähler manifolds $N_{i}$ of dimensions $4 n_{1}$ and $4 n_{2}$. Set $n=n_{1}+n_{2}$. Let $f=\left(f_{1}, f_{2}\right)$ and $N=N_{1} \times N_{2}$. If $(x, \gamma)$ is a nontrivial critical point for the energy function $E$ on $P(M, f)$, then the index $\lambda$ of $E_{* *}$ at $(x, \gamma)$ satisfies

$$
\lambda \geq n-m+1 .
$$


Proof. Let $V \subset T_{(x, \gamma)} P(M, f)$ be the quaternion vector space spanned by parallel vector fields $W$ along $\gamma$ orthogonal to the quaternion line $\dot{\gamma} \wedge I \dot{\gamma} \wedge J \dot{\gamma} \wedge K \dot{\gamma}$ such that $(W(0), W(1)) \in \mathcal{V} \cap f_{*}\left(T_{x} N\right)$. Note that $V$ and $\mathcal{V} \cap f_{*}\left(T_{x} N\right)$ are $\mathbb{H}$-isomorphic, hence by (4.3) we get $\operatorname{dim}_{\mathbb{H}}(V) \geq n-m$.

We claim that in our case $\operatorname{dim}_{\mathbb{H}}(V) \geq n-m+1$.

Indeed, if $x=\left(x_{1}, x_{2}\right)$, it is easy to see that the critical point $(x, \gamma)$ satisfies $\dot{\gamma}(0) \perp\left(f_{1}\right)_{*}\left(T_{x_{1}} N_{1}\right)$ and $\dot{\gamma}(1) \perp\left(f_{2}\right)_{*}\left(T_{x_{2}} N_{2}\right)$. Therefore $(\dot{\gamma}(0), 0),(I \dot{\gamma}(0), 0)$, $(J \dot{\gamma}(0), 0)$ and $(K \dot{\gamma}(0), 0)$ are all orthogonal to $\left(f_{1}\right)_{*}\left(T_{x_{1}} N_{1}\right) \times\left(f_{2}\right)_{*}\left(T_{x_{2}} N_{2}\right)=$ $f_{*}\left(T_{x} N\right)$; similarly $(0, \dot{\gamma}(1)),(0, I \dot{\gamma}(1)),(0, J \dot{\gamma}(1))$ and $(0, K \dot{\gamma}(1))$ are all orthogonal to $f_{*}\left(T_{x} N\right)$. Obviously, all the vectors $(\dot{\gamma}(0), 0),(I \dot{\gamma}(0), 0),(J \dot{\gamma}(0), 0),(K \dot{\gamma}(0), 0)$, and $(0, \dot{\gamma}(1)),(0, I \dot{\gamma}(1)),(0, J \dot{\gamma}(1))$ and $(0, K \dot{\gamma}(1))$ are orthogonal to $\mathcal{V}$. Therefore

$$
\operatorname{dim}_{\mathbb{H}}(V)=\operatorname{dim}_{\mathbb{H}}\left(\mathcal{V} \cap f_{*}\left(T_{x} N\right)\right) \geq n-m+1
$$

Endow $V$ with the inner product $\langle X, Y\rangle:=\langle X(0), Y(0)\rangle$. Consider the real symmetric bilinear form $Q(v, w)=E_{* *}(v, w)$. Note that the index of $E_{* *}$ is not less than the index of $E_{* *}$ restricted to $V$. Therefore by Lemma 4.1 it suffices to prove that for any nontrivial vector $X \in V$ it holds that

$$
Q(X, X)+Q(I X, I X)+Q(J X, J X)+Q(K X, K X)<0,
$$

where $I, J, K$ defines the quaternionic structure on $V$.

By Proposition 1.2 the left side of (4.4) equals to

$$
-\int_{0}^{1} \frac{\mu}{m+2}\|X\|^{2}\|\dot{\gamma}\|^{2}<0
$$

where $\mu$ is the Einstein constant of $M$. The desired result follows.

\section{Proofs of Theorem A}

Let $M$ be a positive quaternionic Kähler manifold. Let $P(M)$ denote the space of $C^{k}$ paths $\gamma:[0,1] \rightarrow M$. Let $\pi: P(M) \rightarrow M \times M$ be the projection to the pair of end points (i.e. $(\gamma(0), \gamma(1))$ ). It is a standard result in topology that $\pi$ is a Serre fibration

$$
\Omega M \rightarrow P(M) \stackrel{\pi}{\rightarrow} M \times M
$$


with fiber the loop space $\Omega M$ with a fixed base point. By definition we know that the projection to the first factor $p: P(M, f) \rightarrow N$ is exactly the pullback fibration by $f: N \rightarrow M \times M$ from $\pi: P(M) \rightarrow M \times M$. In particular, there is a homotopy exact sequence

$$
\cdots \rightarrow \pi_{i}(P(M ; f)) \rightarrow \pi_{i}(N) \rightarrow \pi_{i-1}(\Omega M) \rightarrow \pi_{i-1}(P(M ; f)) \rightarrow \cdots
$$

Proof of Theorem A.

By Lemma 3.3 and Theorem 4.2, (A1) follows.

Note that $\pi_{i-1}(\Omega M)=\pi_{i}(M)$. In particular, $\Omega M$ is path connected since $M$ is simply connected (Theorem 1.1). Then $P(M ; f)$ is path connected. If $n>m+1$, by Theorem 3.5 and Theorem 4.2, $P(M ; f)$ has the homotopy-type of a CW-complex, obtained from $f^{-1}(\Delta)$ by attaching cells of dimensions at least 2 . Thus $f^{-1}(\Delta)$ is path connected. This proves (A2).

Let $p_{1}: P(M ; f) \rightarrow N_{1}$ denote the composition of the bundle projection $p$ with the projection from $N_{1} \times N_{2} \rightarrow N_{1}$ to the first factor. Note that $p_{1}$ is a fibration with fiber $V$, the pullback fibration fitting in the commutative diagram below

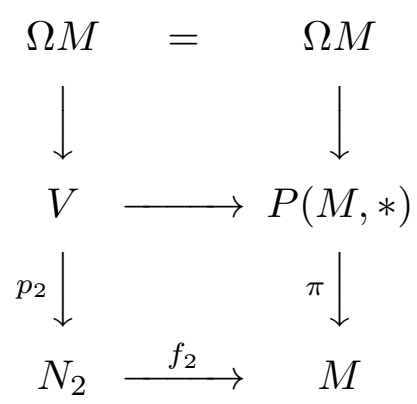

where the right side is the principal path fibration. Note that $P(M, *)$ is contractible. By the above diagram $V$ is the homotopy fiber of the map $f_{2}: N_{2} \rightarrow M$. Therefore by the fibration homotopy exact sequence it follows that

$$
\pi_{i}(V) \approx \pi_{i+1}\left(M, N_{2}\right)
$$

for all $i$. This together with the homotopy exact sequence for the fibration

$$
\begin{gathered}
V \rightarrow P(M ; f) \\
p_{1} \downarrow \\
N_{1} \\
19
\end{gathered}
$$


and the long exact sequence for the map $i_{1}: f^{-1}(\Delta)=N_{1} \cap N_{2} \rightarrow N_{1}$ gives a commutative diagram:

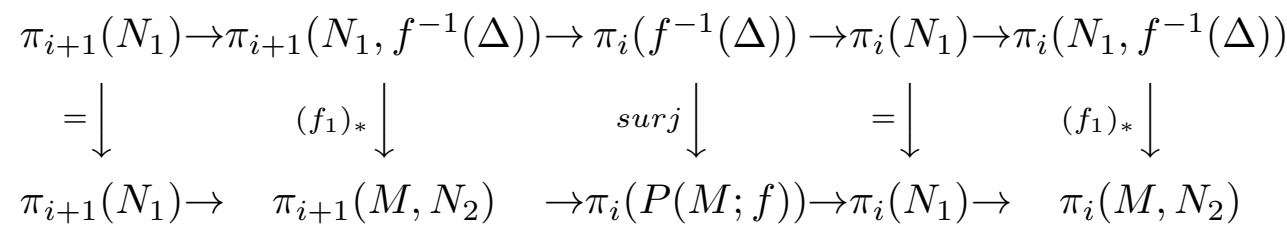

The middle homomorphism is surjective for $i \leq n-m$ since by Theorem 3.5 and Theorem $4.2 \pi_{i}\left(P(M ; f), f^{-1}(\Delta)\right)=0$. From the 5-lemma the commutative diagram above implies that

$$
\left(f_{1}\right)_{*}: \pi_{i}\left(N_{1}, f^{-1}(\Delta)\right) \rightarrow \pi_{i}\left(M, N_{2}\right)
$$

is an isomorphism for all $i \leq n-m$ and a surjection for $i=n-m+1$. The desired result follows.

Proof of Theorem 0.2.

Since $f: N \rightarrow M$ is an immersion, it suffices to show that $f$ is a one-to-one map. Note that $\left.(f, f)^{-1}(\Delta)=\{(x, x), x \in N\} \cup\{(x, y): f(x)=f(y), x \neq y)\right\}$. Hence, if $f$ is not one-to-one, then $f^{-1}(\Delta)$ is not connected; a contradiction to (A2).

\section{REFERENCES}

[Al] D. V. Alekseevsky, Compact quaternion spaces, Functional Anal. Appl. 2 (1968), 106-114.

[AHS] M. Atiyah, N. Hitchin, I.M. Singer, Self-duality in four dimensional Riemannian geometry, Proc. Roy. Soc. London, Ser. A. 362 (1978), 425-461.

[AS] M. Atiyah, I.M. Singer, The index of elliptic operators III, Ann. Math 87 (1968), 546-604.

[Ba] W. Barth, Transplating cohomology classes in complex projective space, Amer. J. Math. 92 (1970), 951-967.

[Be] A. Besse, Einstein manifolds, Berlin Heidelberg New York Springer (1987).

[Ber] M. Berger, Sur les groupes d'holonomie des varietes a connexion affine et des varietes riemanniennes, Bull. Soc. Math. France 83 (1955), 279-330.

[Bi] R. Bielawski, Compact hyperkähler 4n-manifolds with a local tri-Hamiltonian $\mathbb{R}^{n}$-action, Math. Ann. 314 (1999), 505-528.

[Br] G. Bredon, Introduction to compact transformation groups, Academic Press 48 (1972).

[DS] A.Dancer, A.Swann, Quaternionic Kähler manifolds of cohomogeneity one, Internat. J. Math. 10 (1999), 541-570.

[FMR] F. Fang; S. Mendonça; X. Rong, A Connectedness principle in the geometry of positive curvature, preprint.

[Fr] T. Frankel, Manifolds of positive curvature, Pacific J. Math. 11 (1961), 165-174.

[Fuj] A. Fujiki, On the de-Rham cohomology groups of compact Kähler symplectic manifolds, in Algebraic Geometry, Sendai (Advances Studies in Pure Math. 10, T. Oda (ed) North Holland 1987).

[Fu] W. Fulton, On the topology of algebraic varieties, Proc. Symp. in Pure Math. 46 (1987), 15-46. 
[FH] W. Fulton; J. Hansen, A connectedness theorems for projective varieties, with applications to intersections and singularities of mappings, Ann. Math 110 (1979), 159-166.

[FL] W. Fulton; R. Lazarsfeld, Connectivity and Its Applications in Algebraic Geometry, Lecture Notes in Mathematics 862, Springer-Verlag, 26-92.

[Gr1] A. Gray, A note on manifolds whose holonomy is a subsgroup of $S p(n) S p(1)$, Mich. Math. J 16 (1965), 125-128.

[Gr] K. Grove, Geodesics satisfying general boundary conditions, Comment. Math. Helv. (1973), 376-381.

[HS] A. Hattori, H. Tanihuchi, Smooth $S^{1}$-action and bordism, J. Math. Soc. Japan 24 (1972), 701-731.

[HH] H. Herrera, R. Herrera, Â-genus on non-spin manifolds with $S^{1}$ actions and the classification of positive quaternionic Kähler 12-manifolds, J. Diff. Geom. 61 (2002), 341-364.

[Hi] N. Hitchin, Kähler twistor spaces, Proc. London Math. Soc. 43 (1981), 133-150.

[Kr] V. Kraines, Topology of quaternionic Kähler manifolds, Trans. Amer. Math. Soc. 122 (1966), 357-367.

[Le] C. Lebrun, Fano manifolds, contact structures and quaternionic geometry, Inter. J. Math. 6 (1995), 419-437.

[Le-Sa] C. Lebrun, S. Salamon, Strong rigidity of positive quaternionic Kähler manifolds, Invent. Math. 118 (1994), 109-132.

[Le] S. Lefschetz, L'analysis situs et la geometrie algebrique, Gauthier-Villars, Paris (1924).

[Ma] S. Marchiafava, Su alcune sottovareita che ha intersse considerare in una varieta Kaehleriana quaternionale, Rend. Mat. VII 10 (1990), 493-529.

[Mi] J. Milnor, Morse theory, Ann. Math. Stud. Princeton university press (1963).

[NT] T. Nagano, M. Takeuchi, Signature of quaternionic Kähler manifolds, Proc.Japan Acad. 59 (1983), 384-386.

[PV] F.Podesta, L.Verdiani, A note on quaternionic-Kähler manifolds, Internat. J. Math. 11 (2000), 279-283.

[PS] Y.S. Poon, S. Salamon, Eight-dimensional quaternionic Kähler manifolds with positive scalar curvature, J. Diff. Geom. 33 (1991), 363-378.

[Sa] S. Salamon, Quaternionic Kähler manifolds, Invent. Math. 67 (1982), 143-171.

[SW] R. Schoen; J. Wolfson, Theorems of Barth-Lefschetz types and Morse theory on the spaces of paths, Math. Zeit. 229 (1998), 77-89.

[Wo] J.A. Wolf, Complex homogeneous contact structures and quaternionic symmetric spaces, J. Math. Mech. 14 (1965), 1033-1047.

Nankai Institute of Mathematics, Nankai University, Tianjin 300071, P.R.C. Instituto de Matematica, Universidade Federal Fluminense, Niteroi, R.J., Brasil

E-mail address: ffang@nankai.edu.cn ; fuquanfang@eyou.com 